\title{
Exploring Parallel Coordinates Plots in Virtual Reality
}

\author{
Sławomir Konrad Tadeja \\ Timoleon Kipouros

\section{Per Ola Kristensson} \\ Department of Engineering \\ University of Cambridge \\ Cambridge, UK
}

\section{ABSTRACT}

Parallel Coordinates Plots (PCP) are a widely used approach to interactively visualize and analyze multidimensional scientific data in a 2D environment. In this paper, we explore the use of Parallel Coordinates in an immersive Virtual Reality (VR) 3D visualization environment as a means to support the decision-making process in engineering design processes. We evaluate the potential of VR PCP using a formative qualitative study with seven participants. In a task involving 54 points with 29 dimensions per point, we found that participants were able to detect patterns in the dataset compared with a previously published study with two expert users using traditional 2D PCP, which acts as the gold standard for the dataset. The dataset describes the Pareto front for a three-objective aerodynamic design optimization study in turbomachinery.

\section{INTRODUCTION}

As more data is being generated than ever before, researchers from both academia and industry are exploring new techniques for data processing, exploration and effective visualization.A plethora of

Permission to make digital or hard copies of part or all of this work for personal or classroom use is granted without fee provided that copies are not made or distributed for profit or commercial advantage and that copies bear this notice and the full citation on the first page. Copyrights for third-party components of this work must be honored. For all other uses, contact

\section{KEYWORDS} the owner/author(s)

CHI'19 Extended Abstracts, May 4-9, 2019, Glasgow, Scotland UK

Parallel coordinates; virtual reality; data

visualization; visual analytics; decision-making

ACM ISBN 978-1-4503-5971-9/19/05.

https://doi.org/10.1145/3290607.3313068 


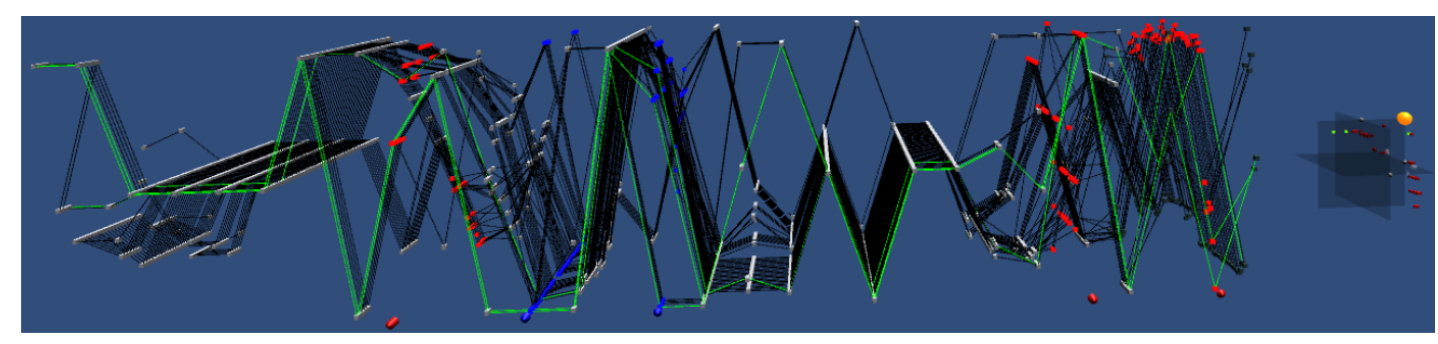

Figure 1: The HMD user's view over the entire PCP visualized in VR. Selected points are highlighted (in light green) across all dimensions. The grey cubes denote individual dimensions while the red color marks the dimension selected across all points visualized on the $3 \mathrm{D}$ scatter plot to the right.

Publications involving Parallel Coordinates Plots: According to Heinrich et al. [7] the number of publications with the term "parallel coordinates" increased from 14 in the year 1991 to approximately 543 in 2011 , to 5620 in 2012, and to 1680000 as reported by Google Scholar on the $8^{\text {th }}$ of September 2018

Dataset: The dataset describes the Pareto front for a three-objective aerodynamic design optimization study in turbomachinery.

Hardware setup: The experimental part of the study was run on a laptop computer with Intel Core i7-7700K CPU Nvidia GeForce GTX1080 GPU, 32GB RAM, working under Windows 10 Pro 64-bit OS. An Oculus Rift was used to immerse users in the VR environment. The interaction was facilitated with the help of an Xbox gamepad controller. methods have been devised to date to process and analyze large and complex volumes of data. Parallel Coordinates Plots (PCP) were introduced by Inselberg [8], and the relevant theory has been developed rigorously since then and presented in great detail by Inselberg [9].

As PCP is so widely used, it was only a matter of time before there would be attempts to recreate PCP visualizations in 3D. There are many ways in which PCP can be visualized in 3D environments using standard computer software, and more recently also in immersive environments, such as virtual reality (VR). Johansson et al. [11] conducted a study that compared existing (at the time) approaches to $3 \mathrm{D}$ visualizations of PCP with a conventional version of $2 \mathrm{D}$ PCP and found that $2 \mathrm{D}$ PCP was the preferred solution. However, while many $3 \mathrm{D}$ versions of $\mathrm{PCP}$ have been explored, only a handful of them have been using truly immersive environments, such as the one provided with today's VR headsets. Even though the 3D setting usually leads to more visual clutter as opposed to the 2D version, the immersiveness component offered by VR may bring additional benefits for the data manipulation and understanding.

\section{RELATED WORK}

Researchers have explored 3D versions of PCP for many years. Recently, there have also been some attempts to use immersive interfaces, such as VR or augmented reality (AR) for 3D PCP visualizations. For example, Butscher et al. [1] introduce the $A R T$ system for collaborative data analysis using AR-based PCP visualization. Their tool usde an AR headset and an additional multitouch table to allow users to interact with the plot. Cordeil et al. [3] presented ImAxes that allows interactive rearrangement of axes according to the user's needs and simultaneously supports $2 \mathrm{D}$ and 3D scatter plots as well. Rosenbaum et al. [16] discusses benefits and challenges of using immersive interfaces for abstract data visualizations and provide an example of Immersive Parallel Coordinates (iPC) [16], 


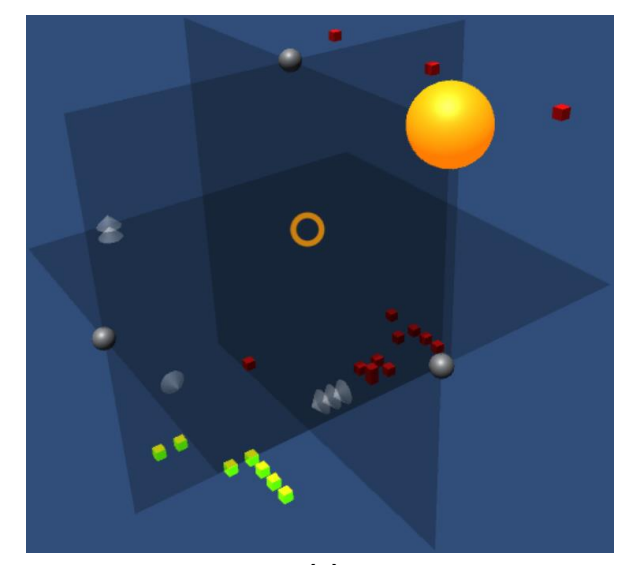

(a)

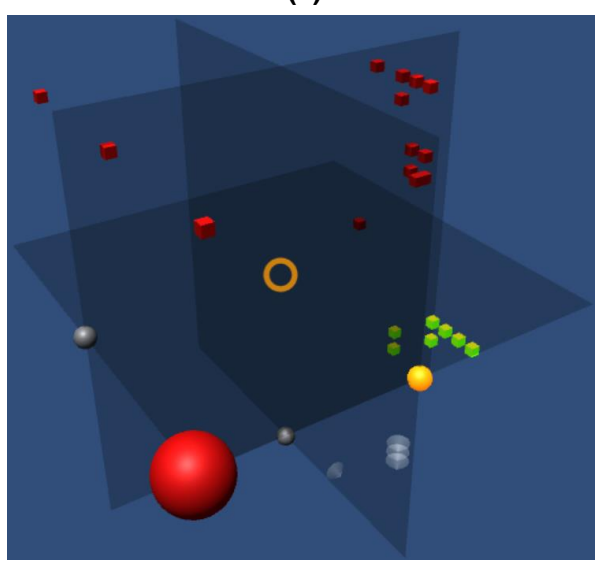

(b)

Figure 2: 3D scatter plot [17] automatically generated out of the selected dimensions. (a) A sample of selected points in the bottom-left corner. The highlighted sphere (in orange) is a movement selector in an active state. (b) The same environment rotated by $90^{\circ}$ along the $X$-axis. which treats the user as part of the visualization itself. Dang et al. [4] proposed a stacking technique that can be used to denote the grouping or clustering of points overlapping with each other due to the similar or identical values they represent using various visualization types. They also present a number of 3D stacked parallel coordinates plots [4]. Moreover, Chang et al. [2] compared PCP with scatter plot matrices (SPLOMs) and their joined side-by-side 2D view and concluded that, for the majority of users and some tasks, the latter brought clear advantages over their individual visualizations.

Our implementation is distinct from prior work in the use of interactive cubes to denote the values for each data item in each dimension. This decision opens a new range of avenues for exploration. For instance, the glyph characteristic [15] can be used to simultaneously show not only the number of values but also other information related to the particular dimensions for a subset of, or even all, data points. Moreover, some of our study participants on their own suggested using a variety of shapes to differentiate between the selected dimensions as relying solely on color might be insufficient.

\section{VISUALIZATION}

To explore PCP in immersive VR we built a visualization system using the Unity game engine. Users interact with the visualizations using a gamepad Xbox controller. In our version of PCP we have given our users the ability to adjust the spatial alignment of their virtual workspace by allowing them to move the elements of the visualization freely in 3D space. In addition, our pattern identification process has been augmented with the use of 3D scatter plots, which are a natural 3D extension of the 2D combined design studied by Chang et al. [2]. The 3D scatter plots provide a suitable clustering solution that allows users to quickly isolate groups of points that are likely to form a pattern. Furthermore, most of the 3D PCP use some form of translucent parallel planes (see $[1,11])$. However, we decided to use a different approach (Figure 1) to simultaneously minimize the possible effects of occlusion and cluttering. Moreover, the adopted Cartesian coordinate system (that is, the units and orientations of the respective axes in 3D space) is the same as for the Unity game engine. Each data item is visualized as a series of identical evenly spaced unit-sized cubes along the $X$-axis connected by lines whose $Y$-coordinates represent values for each dimension scaled to a chosen range. Every point is shifted in the $Z$-axis by a single unit from the previous one, similar to Falkman's [5] Cube package.

The system also allows the user to make a copy of any selected data items, or a grouping of data items, and placed in a different position. The copy of the selected items will be generated in a preconfigured fixed distance towards the direction in which the user is gazing following a tap of a button on the controller. The design and functionality of the interactive 3D scatter plots was borrowed from Tadeja et al. [17]. They can be freely moved in 3D space, rotated along any of the three main axes in $90^{\circ}$ intervals (Figure 2) and have very basic, built-in clustering capabilities (Figure 3). Moreover, all the duplicates, scatter plots and the PCP are interconnected to support the brushing and linking interaction techniques. 


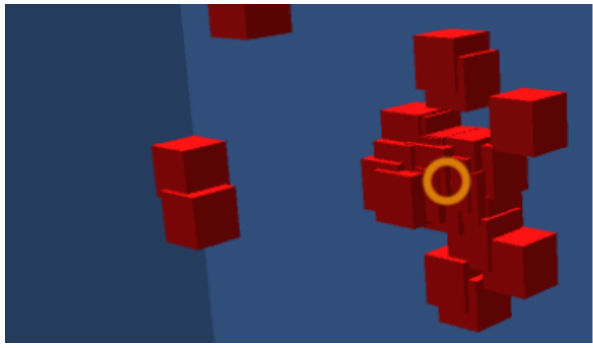

(a)



(b)

Figure 3: Portion of the scatter plot with a number of overlapping points (a). Each group is automatically recognized as a cluster. Selecting any of the points within a group activates all of them as seen in (b) and simultaneously reflects this selection onto the Parallel Coordinates visualization.

\section{OBSERVATIONAL STUDY}

As PCP was designed to deal with complex, multidimensional data, the intended target audience consists of either experts possessing specialized domain knowledge or users that, at the very least, are already familiar with the technique. At this early stage we decided to investigate if there are any advantages in translating PCP to immersive VR and, if there are any potential benefits, which major bottle-necks or challenges arise as a consequence of translating 2D PCP to 3D VR PCP.

As a consequence, this work studies the system's efficacy in an observational study with a group of participants consisting of a mixture of seasoned experts and novice users with regards to conventiona PCP and VR. This method, listed by Lam et al. [14] in their review of common practices for visualization evaluation methods, relies on an observatory approach rather than quantitative methods. To conduct the experiment, we used a dataset from Kipouros et al. [13] that contains 54 data items with 29 dimensions per item split into two categories: design parameters (26) and criteria (3). The seven participants were also asked to self-assess their level of expertise in VR and PCP using a Likert-like scale and they were all pre-screened with the Ishihara's tests for color deficiency [10]. Moreover, they were asked to fill in the simulation sickness questionnaire developed by Kennedy et al. [12] before and after both the training phase and the experiment phase. They were also instructed to stop if they developed any of the sickness symptoms.

The study consisted of three parts: a short oral instruction and presentation of the PCP, its immersive adaptation, and details of all the possible interaction techniques (up to 15 minutes), followed by two experimental sessions, on average 25 minutes for training and 15 minutes in the main study with approximately a 15-minute break in between. The main task was: choose any two (or more) criteria to develop the 3D scatter plot and explore it to identify and select a group of points that potentially represent patterns. Users were also asked to think aloud, that is, discuss what they were doing at the moment and why, what they wished to achieve and what approach/interaction they were trying to use. Some of the interaction elements proved to be challenging for first time users of an Xbox controller and required additional guidance.

\section{RESULTS AND DISCUSSION}

Due to the specific nature of the data used in the study, we were predominantly interested in pattern recognition-(1) which group of points constituted a pattern for the individual participant; and (2) how many of these patterns was an individual participant able to discover. These findings were later on compared with the results gathered using the same dataset from Kipouros et al. [13], in which expert PCP users discovered two patterns containing 11 and 19 data items (which we will from here on denote as patterns $A$ and $B$ respectively) using traditional 2D PCP. Certain levels of variability were expected in the data, hence participants were told to take this variability into consideration. However, the final 


\section{Selected comments:}

"I think compared to Parallel Coordinates before (...) it's better than $2 D$ (..) in that you can see and identify patterns a bit easier."

"If there were more minimaps [scatter plots] $I$ don't know if I could process (...) I would get confused"

"That was (...) other side of Parallel Coordinates that I've never seen."

"I think the initial training session should be longer (...) especially for people not familiar with $V R$ "

“(...) is quite immersive. You don't really feel the outside (...) as soon as you get the feel of the controls it's quite natural. (...) you look around and you identify where you put your previous selection, patterns and so on."

"With the little experience that I have [with 2D] this was more intuitive"

\section{ACKNOWLEDGMENTS}

This work is supported by the EPSRC-1788814 and the Cambridge European \& Trinity Hall Studentships. decision of what constituted a pattern was a decision left to the individual participant. Overall, in some cases, participants were very close to, or successfully identified the complete pattern $A(82 \%$, $82 \%, 72 \%$ and $54 \%$ for the four participants that identified pattern $A)$ or $B(100 \%, 84 \%, 84 \%, 79 \%$ and $79 \%$ for the five participants that identified pattern $B$ ).

These differences likely stem from unfamiliarity with the underlying dataset, and in turn influenced their assessment decision with respect to how much variability was allowed between the values to be considered as noise and not a meaningful value change. The results indicate that it is possible to obtain satisfactory results using our implementation of VR-based PCP on a real-world dataset.

User feedback concentrated on three key aspects of the system: PCP, scatter plots and interaction. Participants seemed to quickly understand which elements constituted a single data item or item group and they also quickly understood the PCP visualization itself. With regard to scatter plots, users indicated a preference for fewer plots in order to limit confusion in their analysis.

To improve clarity, in subsequent iterations of the system we signal to the user which exact dimensions on the PCP were mapped to which axes using textual labels. The scatter plot rotation seems to be a feature not favored by any of the participants. However, it may be due to the datase and bias developed during the training exercise. The simple clustering algorithm has proven to be surprisingly useful even if some of the participants said they could not understand it. Differentiating which object in the cluster users want to interact with may be solved by supporting zooming-in into the cluster structure, or by using a more sophisticated clustering algorithm.

Comments regarding the interaction suggest that participants quickly acquainted themselves with the implemented movement and maneuvering techniques. When using the gamepad, however, a few users reported some difficulty. Hence, it may be necessary to either support fewer features or extend the training session. Distal or cluttered object selection could be augmented with a target acquisition aid (e.g., Bubble Cursor, Grossman et al. [6]).

\section{CONCLUSIONS}

The results of the study revealed that the idea of having a useful 3D PCP cannot yet be completely discarded. This formative qualitative study indicates that VR PCP may be beneficial, or at the very least, is not obviously detrimental to performance compared with 2D PCP. However, the small-scale of the study and the fact that participants were not exposed to a standard 2D PCP interface means it is not possible to conclude that VR PCP would be necessarily more beneficial than 2D PCP. We conjecture that the differences between $\mathrm{VR}$ and $2 \mathrm{D}$ PCP are rather nuanced and most likely there are certain advantages and disadvantages induced by either modality. Future work includes unravelling these nuances in a set of controlled experiments. 


\section{REFERENCES}

[1] Simon Butscher, Sebastian Hubenschmid, Jens Müller, Johannes Fuchs, and Harald Reiterer. 2018. Clusters, Trends, and Outliers: How Immersive Technologies Can Facilitate the Collaborative Analysis of Multidimensional Data. In Proceedings of the 2018 CHI Conference on Human Factors in Computing Systems (CHI '18). ACM, 90:1-90:12. https: //doi.org/10.1145/3173574.3173664

[2] Chunlei Chang, Tim Dwyer, and Kim Marriott. 2018. An Evaluation of Perceptually Complementary Views for Multivariate Data. In 2018 IEEE Pacific Visualization Symposium (PacificVis). 195-204. https://doi.org/10.1109/PacificVis.2018.00033

[3] Maxime Cordeil, Andrew Cunningham, Tim Dwyer, Bruce H. Thomas, and Kim Marriott. 2017. ImAxes: Immersive Axes As Embodied Affordances for Interactive Multivariate Data Visualisation. In Proceedings of the 30th Annual ACM Symposium on User Interface Software and Technology (UIST '17). ACM, 71-83. https://doi.org/10.1145/3126594.3126613

[4] Tuan N. Dang, Leland Wilkinson, and Anushka Anand. 2010. Stacking Graphic Elements to Avoid Over-Plotting. IEEE Transactions on Visualization and Computer Graphics 16, 6 (Nov. 2010), 1044-1052. https://doi.org/10.1109/TVCG.2010.197

[5] Göran Falkman. 2001. Information visualisation in clinical Odontology: multidimensional analysis and interactive data exploration. Artificial Intelligence in Medicine 22, 2 (May 2001), 133-158. https://doi.org/10.1016/S0933-3657(00)00104-4

[6] Tovi Grossman and Ravin Balakrishnan. 2005. The Bubble Cursor: Enhancing Target Acquisition by Dynamic Resizing of the Cursor's Activation Area. In Proceedings of the SIGCHI Conference on Human Factors in Computing Systems (CHI '05). ACM, 281-290. https://doi.org/10.1145/1054972.1055012 event-place: Portland, Oregon, USA.

[7] Julian Heinrich and Daniel Weiskopf. 2013. State of the art of Parallel Coordinates. Eurographics (2013), 95-116.

[8] Alfred Inselberg. 1985. The plane with parallel coordinates. The Visual Computer 1, 2 (Aug. 1985), 69-91. https: //doi.org/10.1007/BF01898350

[9] Alfred Inselberg. 2009. Parallel Coordinates: Visual Multidimensional Geometry and Its Applications. Springer, NY, USA.

10] Shinobu Ishihara. 2017. Ishihara's Tests for Colour Deficiency (38 plates edition ed.). Kanehara Trading Inc, Tokyo, Japan.

[11] Jimmy Johansson, Camilla Forsell, and Matthew Cooper. 2014. On the usability of three-dimensional display in paralle coordinates: Evaluating the efficiency of identifying two-dimensional relationships. Information Visualization 13, 1 (Jan. 2014), 29-41. https://doi.org/10.1177/1473871613477091

[12] Robert S. Kennedy, Norman E. Lane, Kevin S. Berbaum, and Michael G. Lilienthal. 1993. Simulator Sickness Questionnaire: An Enhanced Method for Quantifying Simulator Sickness. The International Journal of Aviation Psychology 3, 3 (July 1993), 203-220. https://doi.org/10.1207/s15327108ijap0303_3

[13] Timoleon Kipouros, Alfred Inselberg, Geoffrey T. Parks, and Mark Savill. 2013. Parallel Coordinates in Computationa Engineering Design. In AIAA Multidisciplinary Design Optimization Specialists, Vol. 1750. https://doi.org/10.2514/6 2013- 1750

[14] Heidi Lam, Enrico Bertini, Petra Isenberg, Catherine Plaisant, and Sheelagh Carpendale. 2012. Empirical Studies in Information Visualization: Seven Scenarios. IEEE Transactions on Visualization and Computer Graphics 18, 9 (Sept. 2012), 1520-1536. https://doi.org/10.1109/TVCG.2011.279

[15] William Ribarsky, Jay Bolter, Augusto Op Den Bosch, and Ron Van Teylingen. 1994. Visualization and Analysis Using Virtual Reality. Computer Graphics and Applications, IEEE 14 (Feb. 1994), 10-12. https://doi.org/10.1109/38.250911

[16] René Rosenbaum, Jeremy Bottleson, Zhuiguang Liu, and Bernd Hamann. 2011. Involve Me and I Will Understand! Abstract Data Visualization in Immersive Environments. In Proceedings of the 7th International Conference on Advances in Visual Computing - Volume Part I (ISVC'11). Springer-Verlag, Berlin, Heidelberg, 530-540. http://dl.acm.org/citation.cfm? id $=2045110.2045168$

[17] Slawomir K. Tadeja, Pranay Seshadri, and Per Ola Kristensson. 2019. Exploring Aerospace Design in Virtual Reality with Dimension Reduction. In Proceedings of AIAA SciTech Forum and Exposition. https://doi.org/10.2514/6.2019-2206 